\title{
Augmented thermal performance in a non-uniform heat flux circular tube with twisted tape insert using hybrid nanofluid
}

\author{
Arvind Kumar $^{1}$, Kunal Dey ${ }^{2}$, Suvanjan Bhattacharyya ${ }^{3,}{ }^{*}$, Akshoy Ranjan Paul $^{1}$, Ali Cemal Benim ${ }^{4}$, Devendra Kumar \\ Vishwakarma $^{3}$, Zhongjie Huan ${ }^{5}$ \\ ${ }^{1}$ Department of Applied Mechanics, Motilal Nehru National Institute of Technology Allahabad, Prayagraj, UP, India. \\ ${ }^{2}$ Department of Mechanical Engineering, National Institute of Technology Agartala, Agartala, India. \\ ${ }^{3}$ Department of Mechanical Engineering, Birla Institute of Technology and Science Pilani, Pilani Campus, Vidya Vihar, \\ Rajasthan- 333031, India. \\ ${ }^{4}$ Center of Flow Simulation (CFS), Department of Mechanical and Process Engineering, Duesseldorf University of Applied Sciences, \\ D-40476 Duesseldorf, Germany. \\ ${ }^{5}$ Department of Mechanical Engineering, Mechatronics and Industrial Design, Tshwane University of Technology, Private Bag \\ X860, Pretoria 0001, South Africa.
}

\begin{abstract}
The influence of non-uniform heat transfer on a circular tube with a twisted tape insert using nanofluid (NF) is examined. The circular tube had an inner diameter $20 \mathrm{~mm}$, with $0.5 \mathrm{~mm}$ thickness and 2 $\mathrm{m}$ of length. Wall heat flux conditions were examined for Reynolds number ranging from 5000 to 25000 . Heat flux distribution included partial heating at different circumferential positions. Water was used as a base fluid, while single and multi-nano particles are used for simple and hybrid nanofluids (HNF). The goal of this study is to augment the thermal performance by incorporating non-uniform heating, using a twisted tape insert and by using nanoparticle of different volume fraction. NF act as a fluid additive and twisted tape act as a turbulence promoter and they enhance the heat transfer rate. However, major disadvantage in this investigation is the pressure drop incurred due to the twisted tape and NFs. Hence, a series of simulation are carried out to find out the optimum configuration of the set-up for which heat transfer will be enhanced with minimum pressure drop.
\end{abstract}

\section{Introduction}

Thermal performance of solar collectors can be passively augmented using fluid additives viz. nanofluids (NF) and hybrid nanofluids [1,2] and insertion of turbulence promoters, like guide vanes, wavy strips [3], turbulators [4], roughness element and twisted tape [5]. Conventional fluids possess very low thermal conductivity for enhancing the performance of any thermal system. Choi [6] introduced the NF having the higher thermal conductivity than the conventional fluid. Thermophysical properties of NFs depend on the volume fraction of nanoparticle [7]. Industrial applications of NFs include aerospace, nuclear power, microchips, solar system, and biomedical devices [810]. Hybrid NF demonstrated higher thermal conductivity characteristics than the normal NF. However, proper selection of nanoparticle is a major challenge for the preparation of hybrid NF [11].

On the other hand, a few researchers [12-14] investigated the effects of dimples and perforations on the twisted tapes inserted in the pipes under constant heat flux and found that these special twisted tapes contributed higher thermal performance than the plain twisted tapes.

A group of researchers [15-17] in turbulent flow regime studied non-uniform heating over the circular pipes. Dirker et al. [17] experimentally investigated the influence of non-uniform heating (circumferential heating) on a horizontal circular tube with different heat flux distribution over the outer wall of tube. They set up eight heated segments over the circular tube and found that higher thermal performance was ensured at full segment heating than any other cases. It was noteworthy that the bottom part heating provided the higher average Nusselt number as compared to the other cases except the full segment heating.

From these mentioned works, numerical investigation on non-uniform heating cases has been less noted. For circumferential heat flux distribution on solar collector in a turbulent regime, the effect of nonuniform heating on thermal performance is still unclear. The effective technique among the twisted tape inserts, nanofluid and hybrid nanofluid applicable to the solar collector in terms of thermal performance has not yet been identified.

The intention of the numerical study is therefore, to determine the optimum configuration of the set-up for which heat transfer will be enhanced at low pressure drop with using the non-uniform heating, twisted tape insert, nanofluids and hybrid nanofluids. This numerical study is done on the fully turbulent regime at $\mathrm{Re}=5000-25000$ with circumferential heating (nonuniform heating) using different configuration of fluids such as water (as base fluid), nanofluids (base fluid 
with nanoparticle) and hybrid nanofluids (base fluid with two types of nanoparticles).

\section{Analysis and modelling}

A three-dimensional thermo-fluid dynamics study of a circular tube with twisted tape insert was done via CFD simulations using ANSYS Fluent 19.2 The geometry of the circular tube with twisted tape insert was generated using the ANSYS Design Modeller while the grid is generated using the ICEM-CFD. A grid selection test was performed and 1,777325 cell was found as an optimum meshing size.

\subsection{Geometry}

Fig. 1 shows the geometry of the three-dimensional computational domain. This numerical investigation study carries out on circular tube with twisted tape insert with inner diameter of $20 \mathrm{~mm}$ and $2500 \mathrm{~mm}$ length. For this study the thickness and width of the simple twisted tape is $1 \mathrm{~mm}$ and $16 \mathrm{~mm}$ with twist ratio $\mathrm{y} / \mathrm{W}=3.6$.

Grid independence result of plane channel with uniform heat flux at Reynolds number 1000 shown in Fig. 2. The generated good quality mesh is depicted in Fig. 3. As already mentioned above, a mesh consistent of $1,777,325$ cells is used for the present computation.

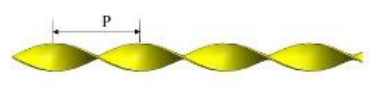

Twisted Tape Cross Sectional Area:
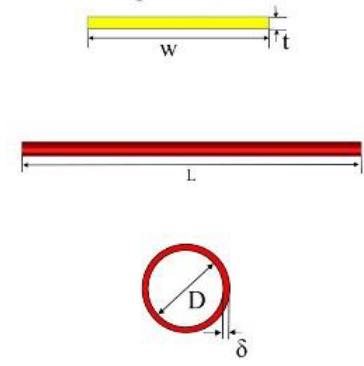

Fig. 1. Schematic diagram of computational domain

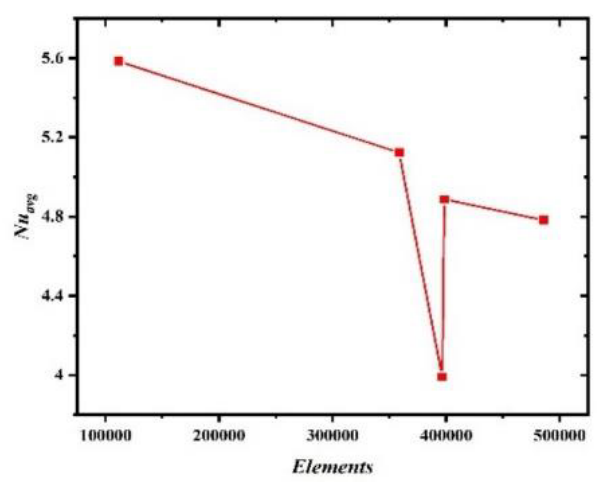

Fig. 2. Grid independence result of plane channel with uniform heat flux $=1000 \mathrm{~W} / \mathrm{m}^{2}$ at $\mathrm{Re}=1000$.

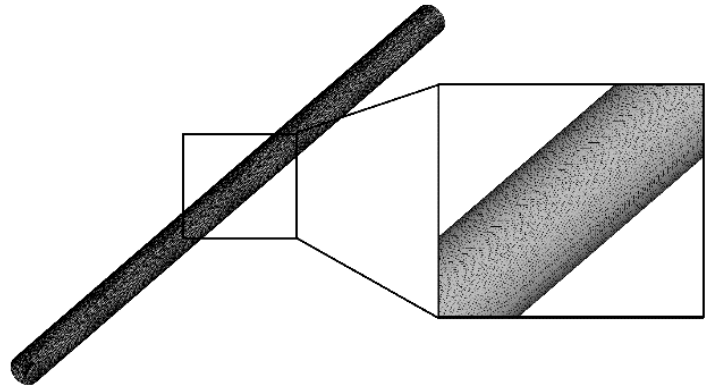

Fig. 3. Meshing for the domain.

The non-uniform heat flux distribution is shown in Fig. 4. The heat flux on heated section is $1000 \mathrm{~W} / \mathrm{m}^{2}$ and the unheated section is taken as insulated. For Case A only one section was heated with $1000 \mathrm{~W} / \mathrm{m}^{2}$ and other section was insulated. For Case B two section was heated with $1000 \mathrm{~W} / \mathrm{m}^{2}$ and rest of the two section was taken as insulated. For Case $\mathrm{C}$ three section heated with $1000 \mathrm{~W} / \mathrm{m}^{2}$ and one section is insulated. For Case $\mathrm{D}$ all section is heated with $1000 \mathrm{~W} / \mathrm{m}^{2}$.

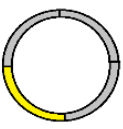

A

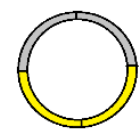

B

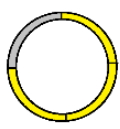

$\mathrm{C}$

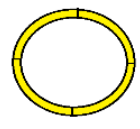

$\mathrm{D}$
Insulated Face

Heated Face

Fig. 4. Heat flux distribution

\subsection{Governing Equations}

In the current study, it is assumed that the flow through the circular tube incompressible, turbulent and statistically steady. Differential equations of mass, momentum and energy conservation under the present assumptions provided below (the time-averaged equations solved for the turbulent flow, and the equations of the turbulence model are not provided for simplicity)

Continuity Equation:

$$
\nabla \cdot \vec{V}=0
$$

Momentum Equation:

$$
\rho(\vec{V} \cdot \nabla) \vec{V}=-\nabla p+\mu \nabla^{2} \vec{V}+\rho \vec{g}
$$

$$
\begin{aligned}
& \text { Energy Equation: } \\
& \qquad \vec{V} \cdot\left(\vec{V}\left(\rho C_{p} T\right)\right)=\nabla \cdot(k \nabla T)
\end{aligned}
$$

where, $\vec{V}, p, T, g, \rho, \mu, k, C_{p}$ denote the velocity vector, pressure, temperature, gravitational acceleration, density, molecular viscosity, molecular thermal conductivity and isobaric specific heat capacity, respectively. 


\subsection{Thermo-physical properties of nanofluids and hybrid nanofluids}

The thermophysical properties of nanofluids and hybrid nanofluids such as density, specific heat capacity, thermal conductivity, and viscosity have been calculated by using the following equations. [1]. For the nanofluid, these are termed as "effective" below.

\subsubsection{Effective density}

For calculating the effective density of nanofluid the Pak and Cho [18] model is used:

$$
\rho_{e f f}=(1-\varphi) \rho_{b f}+\varphi \rho_{n p}
$$

For calculating the effective density of hybrid nanofluid Takabi and Salehi [19] model is used :

$$
\begin{aligned}
\left.\rho_{e f f}\right)_{y b r i d}= & \varphi_{n p 1} \rho_{n p 1}+\varphi_{n p 2} \rho_{n p 2} \\
& +(1-\varphi) \rho_{b f}
\end{aligned}
$$

\subsubsection{Effective specific heat}

For calculating the effective specific heat of nanofluid Pak and Cho [18] model is used :

$$
C_{p, e f f}=\frac{(1-\varphi)\left(\rho C_{p}\right)_{b f}+\varphi \cdot\left(C_{p} \cdot \rho\right)_{n p}}{\rho_{e f f}}
$$

For calculating the effective specific heat of hybrid nanofluid Takabi and Salehi [19] model is used.

$$
=\frac{\left.C_{p, e f f}\right)_{h y b r i d}}{(1-\varphi) \rho_{b f} C_{p, b f}+\varphi_{n p 1} \rho_{n p 1} C_{p, n p 1}+\varphi_{n p 2} \rho_{n p 2} C_{p, n p 2}}
$$

\subsubsection{Effective thermal conductivity}

For estimating the effective thermal conductivity of nanofluid the temperature dependent model by Chon et al. [20], is used for $d_{b f}=0.384 \mathrm{~nm}$ and $d_{n p}=47 \mathrm{~nm}$.

$$
\begin{aligned}
& \frac{k_{e f f}}{k_{b f}} \\
& =1 \\
& +64.7(\varphi)^{0.764}\left(\frac{d_{b f}}{d_{n f}}\right)^{0.369}\left(\frac{k_{b f}}{k_{n f}}\right)^{0.7476} \operatorname{Pr}_{T}^{0.9955} \operatorname{Re}^{1.231}
\end{aligned}
$$

For estimating the effective thermal conductivity of hybrid nanofluid the Maxwell model [21] is used :

$$
\frac{k_{e f f}}{k_{b f}}=\left[\frac{\left(k_{n p}+2 k_{b f}\right)-2 \varphi\left(k_{b f}-k_{n f}\right)}{\left(k_{n p}+2 k_{b f}\right)+\varphi\left(k_{b f}-k_{n f}\right)}\right]
$$

\subsubsection{Effective viscosity}

For estimating the effective viscosity of nanofluid temperature dependent Corcione model [22] is used :

$$
\frac{\mu_{e f f}}{\mu_{b f}}=\frac{1}{\left(1-34.87\left(\frac{d_{n p}}{d_{b f}}\right)^{-0.3} \varphi^{1.03}\right)}
$$

For estimating the effective viscosity of hybrid nanofluid Pak and Cho [18] model is used:

$$
\frac{\mu_{e f f}}{\mu_{b f}}=\left(1+39.11 \varphi+533.9 \varphi^{2}\right)
$$

\subsection{Numerical Approach}

The finite volume based CFD solver ANSYS Fluent 19.2 was employed to solve this present problem. The governing equations are discretized by the finite volume method. The SST k- $\omega$ Model was used as the turbulence model. The SIMPLE pressure-velocity coupling scheme was used. A second-order upwind scheme was used for momentum, energy, turbulent kinetic energy and turbulent specific dissipation rate equations. Convergence criteria for continuity, momentum and energy equation are set to $10^{-5}, 10^{-5}$ and $10^{-7}$, respectively.

\section{Results and discussion}

\subsection{Validation}

Fig. 5 (a) and (b) show the validation of Nusselt number and friction factor, respectively. The Nusselt number for water for plain smooth channel is compared with correlations of the Notter and Sleicher [23] and Pak and Cho [18]. A satisfactory agreement is found between present study results and the correlations. For validation of friction factor, Blasius correlation [24] is employed. A very good agreement between present study and values obtained from correlation is observed. $\pm 5 \%$ deviation in the Nusselt number and $\pm 2 \%$ deviation in the friction factor is observed, which show that the present model is reliable.

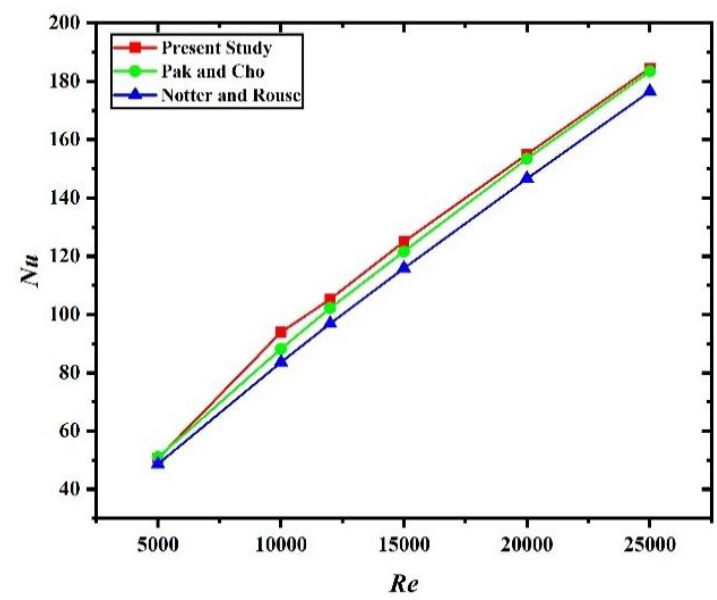

Fig. 5(a). Validation: Nusselt number 


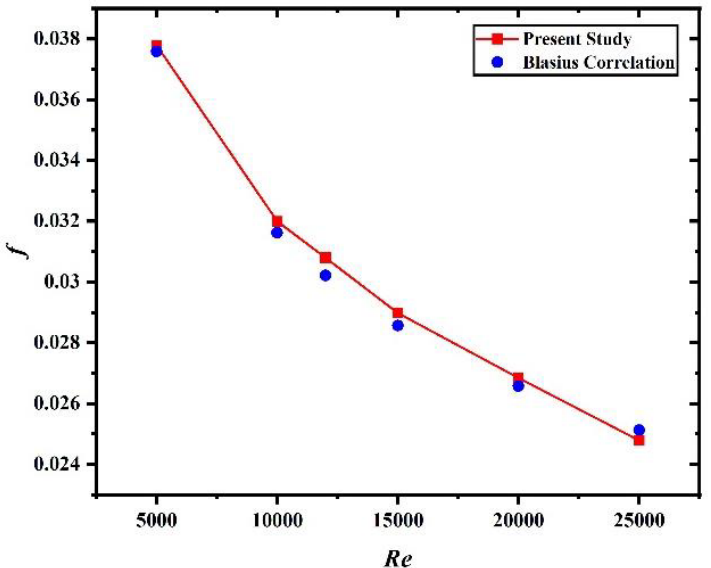

Fig. 5(b). Validation : Friction factor

\subsection{Heat Transfer}

The average Nusselt number was calculated for all the cases with all volume concentrations of nanofluid and hybrid nanofluid. The value of average heat transfer coefficient is increasing with the increase in the volume concentration of nanoparticle in nanofluid and hybrid nanofluid. The hybrid nanofluid having higher thermal conductivity than the nanofluid that leads to increase in the thermal performance of the circular tube which is used for this study. The average Nusselt number was calculated by taking the average over the wall surfaces. The average Nusselt number is defined as $(\mathrm{Nu})_{\text {avg }}$ [25-28].

$$
N u_{\text {avg }}=\frac{a v \cdot D}{K}
$$

The average Nusselt number was increasing with the increase in the Reynolds number. For twisted tape with hybrid nanofluid with $3 \%$ concentration having the highest average Nusselt number than the other cases (Fig. 6 (a) and Fig. 6 (b)).

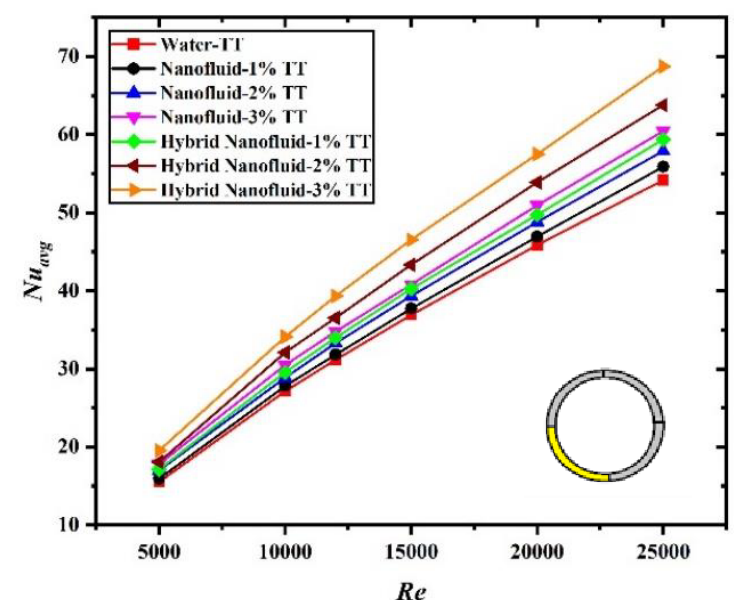

(a)

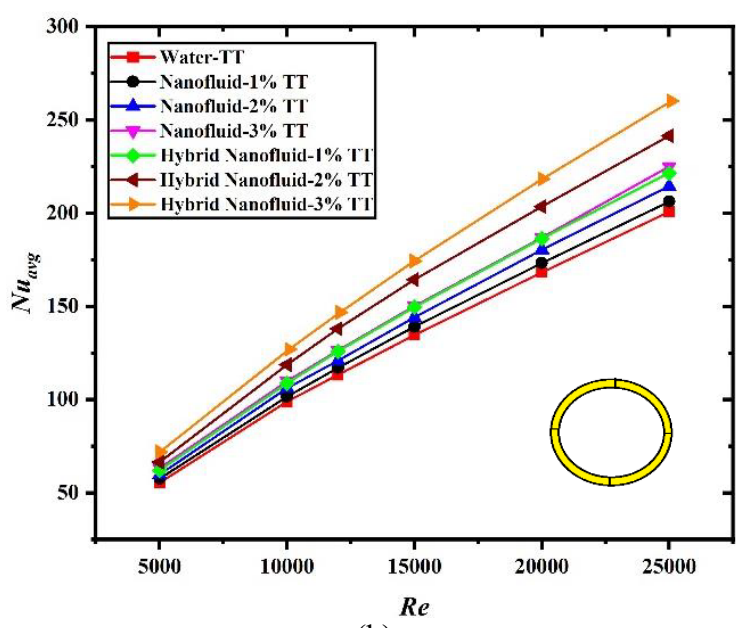

(b)

Fig. 6. Nusselt number for tube with twisted tape (a) Face 1 Heated (b) Face 1, 2, 3 and 4 Heated.

Fig. 7 shows the Nusselt number as a function of Reynolds number for $3 \%$ volume concentration of hybrid nanofluids for case A, B, C and D. It was clear from the figure that for case $\mathrm{D}$, the Nusselt number is highest followed by $\mathrm{C}, \mathrm{B}$ and $\mathrm{A}$, respectively. A maximum of 4 times enhancement is clearly visible between case $\mathrm{D}$ and case $\mathrm{A}$. The uniformly heating of all faces results in better mixing of fluids near the wall with the fluid at the core which led to higher Nusselt number.

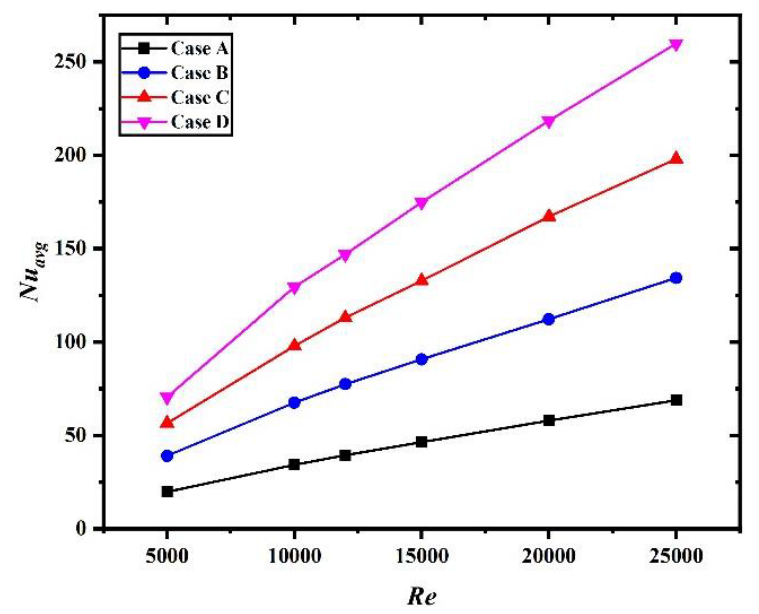

Fig. 7. Comparison of Nusselt number for $3 \%$ volume concentration hybrid nanofluid.

\subsection{Pressure Drop}

Fig. 8 shows the friction factor as a function of Reynolds number and it is clear from figure that the pressure drop is increasing with the increase in Reynolds number as well as increase in volume concentration of nanoparticle of nanofluid and hybrid nanofluid. Minimum pressure drop was noted in the plane channel without twisted tape insert. Maximum pressure drop was noted for the case of circular tube with twisted insert using hybrid nanofluid with $3 \%$ volume concentration of nanoparticle. 


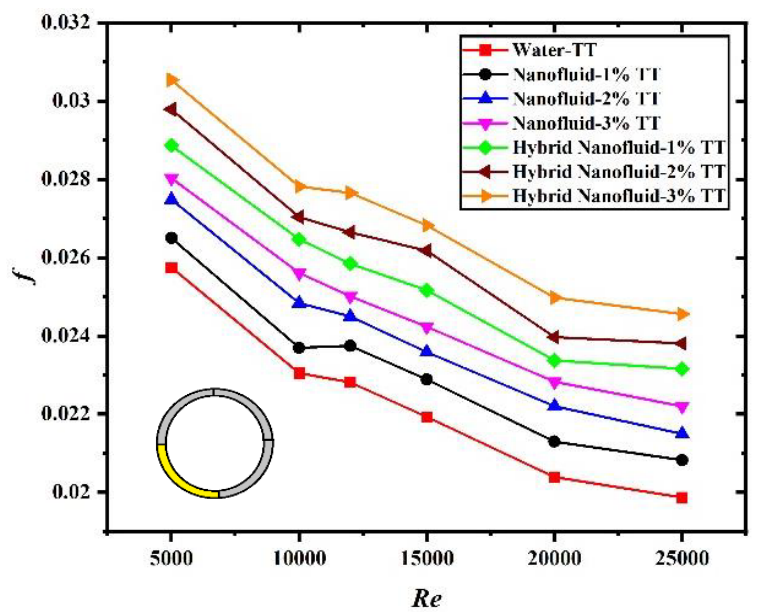

Fig. 8. Friction factor for tube with twisted tape for Face 1 heated.

Fig. 9 shows the friction factor as a function of Reynolds number for 3\% volume concentration hybrid nanofluids for case $\mathrm{A}, \mathrm{B}, \mathrm{C}$ and $\mathrm{D}$. It was clear from the figure that for case $\mathrm{D}$, the friction factor is highest followed by $\mathrm{C}, \mathrm{B}$ and $\mathrm{A}$, respectively. The higher volume concentration of nanoparticles makes the fluids more viscous due to which flow resistance increases which result into higher friction factor. A maximum $26 \%$ increase in the friction factor has been noticed for the case $\mathrm{D}$ when compared with the case A result.

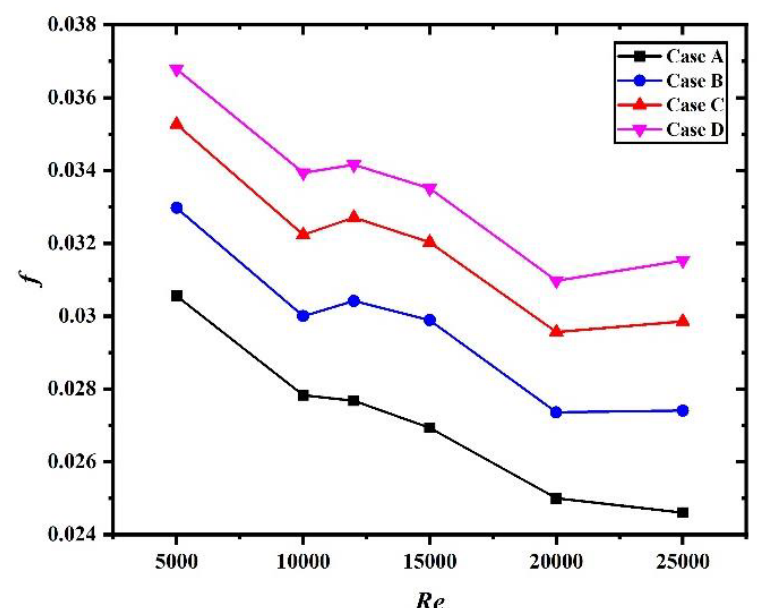

Fig. 9. Comparison of friction factor for $3 \%$ volume concentration hybrid nanofluid.

\section{Conclusion}

In this numerical study of augmented thermal performance in non-uniform heat flux circular tube with twisted tape insert using nanofluids has been implemented and the results in terms of the average Nusselt number and friction factor have been validated with the correlations.

Nusselt number increases with the increase of Reynolds number for all the tested cases. Hybrid nanofluid enhancing more heat transfer than the single acting nanofluid. Hybrid nanofluid with twisted tape inserts has the highest heat transfer than the single acting nanofluid and base fluid.
It is also found that the pressure drop is maximum for the case of hybrid nanofluid (3\% volume concentration $\mathrm{Al}_{2} \mathrm{O}_{3}+\mathrm{Ag}$ ) and it increases with the increase in Reynolds number.

The authors gratefully acknowledge the International cooperation division and India South Africa Bilateral Cooperation Scheme (DST/IBCD/SA/2021/5) for the financial support.

\section{References}

1. S. K. Singh, S. Bhattacharyya, A. R. Paul, M. Sharifpur, J. P. Meyer, in Math. Methods Appl. Sci. (2020), DOI:10.1002/mma.6849

2. M. S. Tahat, A. C. Benim, Defect and Diffusion Forum 374, 148 (2017)

3. S. Bhattacharyya, H. Chattopadhyay, A. C. Benim, Procedia Eng., 157, 19 (2016)

4. S. Bhattacharyya, H. Chattopadhyay, A. Guin, A. C. Benim, Heat Transf. Eng., 40, 1451 (2019)

5. S. K. Saha, S. Bhattacharyya, P. K. Pal, Exp. Therm. Fluid Sci., 41, 121 (2012)

6. S. U. S. Choi, FED 231, 99 (ASME, New York, 1995)

7. P. K. Nagarajan, J. Subramani, S. Suyambazhahan, R. Sathyamurthy, Energy Procedia, 61, 2416 (2014)

8. S. Bhattacharyya, H. Chattopadhyay, A. C. Benim, Prog. Comput. Fluid Dyn., 17, 390 (2017)

9. S. Bhattacharyya, J. Therm. Anal. Calorim., 140, 1355 (2020)

10. S. Bhattacharyya, A. C. Benim, R. Bennacer, K. Dey, Heat Transf. Eng. (2021) DOI: 10.1080/01457632.2021.1875168

11. P. K. Das, J. Mol. Liq., 240, (2017)

12. S. Bhattacharyya, H. Raghvanderan B., A. R. Paul, Exp. Heat Transf., 34, 493 (2021)

13. T. Dagdevir, V. Ozceyhan, Int. J. Therm. Sci., 159, 106564 (2021)

14. S. Bhattacharyya, A. C. Benim, H. Chattopadhyay, A. Banerjee, Exp. Heat Transf., 32, 411 (2019)

15. Z. Huang, Z. Y. Li, W. Q. Tao, Appl. Energy, 185, 2194 (2017)

16. X. W. Zhu, J. Q. Zhao, L. Zhu, Appl. Therm. Eng., 103, (2016)

17. J. Dirker, J. P. Meyer, W. J. Reid, Exp. Therm. Fluid Sci., 98, (2018)

18. B. C. Pak, Y. I. Cho, Exp. Heat Transf., 11, 151 (1998)

19. B. Takabi, S. Salehi, Adv. Mech. Eng., 2014, 1, (2014)

20. C. H. Chon, K. D. Kihm, S. P. Lee, S. U. S. Choi, Appl. Phys. Lett., 87, 1 (2005)

21. M. James Clerk, A Treatise on Electricity and Magnetism (Cambridge University Press, Cambridge, 2010) 
22. M. Corcione, Energy Convers. Manag., 52, 789 (2011)

23. R. H. Notter, C. A. Sleicher, Chem. Eng. Sci., 27, 2073 (1972)

24. H. Blasius, in Mitteilungen Über Forschungsarbeiten Auf Dem Gebiete Des Ingenieurwesens (1913), pp. 1-41

25. S. Bhattacharyya, M. Pathak, M. Sharifpur, S. Chamoli, D. R. E. Ewim, J. Therm. Anal. Calorim., 145, 1019 (2021)

26. S. Bhattacharyya, H. Raghvanderan B., A. R. Paul, Exp. Heat Transf. 1 (2020)

27. S. Bhattacharyya, A. Roy, H. Chattopadhyay, A. Rakshit, in: Recent Adv. Chem. Eng. (Springer, Berlin, 2016) pp.11.

28. S. Bhattacharyya, H. Chattopadhyay, A. Swami, M. K. Uddin, Int. J. Heat Technol., 34, 727 (2016) 